\title{
Hybrid Object Detection and Image Recovery Techniques
}

\author{
Upasana Sharma \\ Department of Software Engineering \\ RGPV University \\ Bhopal, INDIA
}

\author{
Vineet Richariya, PhD \\ Department of Computer Science \& Engineering \\ RGPV University \\ Bhopal, INDIA
}

\begin{abstract}
With the change of time arise the need to work on image processing. This paper gives work on two aspects of image. One Recovery or reconstruction of image and at the other end it is for object detection. This paper investigates the possibility of reconstruction of images. This paper is concerned with a removing corruption of an image and remove problem in image. We propose a new algorithm for solving one of the image restorations and reconstruction algorithm. At another end we also concentrate on object detection. The principal component of image analysis technique is image matching. This principal component became of extreme importance in different area of applications such as in navigation, guidance, automatic surveillance, robot vision. Experiments on a set of image restoration and reconstruction along with object detection show that proposed algorithm performs well enough.
\end{abstract}

\section{Keywords}

Image fusion, Image Restoration, Image Registration, Object Detection, Image Processing, Digital Image.

\section{INTRODUCTION}

The area of image processing is one such area of signal processing where the input for processing is a digital image for example photograph and video frames; and the processed output is either the image or some parameters relating to the digital input image [6,8]. A large number of image processing methods taken a two dimension consideration of image and applied different methods of signal processing over it.

\section{Digital Image Processing:}

- Human vision is the ability which enables ones to become aware and conscious about ones surrounding.

- The ramifications of human vision are electronically understood and duplicate to obtain the computer vision.

- This is not an easy job to provide vision to the computer system- as the real world is a three dimensional space (3D)and when computer system analyze the different object existing in three dimensional world, the different sensors capture two dimensional information of the object, because of this projection to lower dimensional incurred a considerable loss of information

- The job of understanding computer vision can be simplify by distinguishing the two level i.e lower level processing and higher level processing.

- However in the method of lower level processing the used knowledge about the image content is low.
- On other part the higher level of processing utilizes advance knowledge, goals and different proposed plans helps in achieving goals. One popular method called Artificial Intelligence (AI) applied in many cases. In this level of processing computer vision enables to emulate the human intellectual and obtained information from the image helps in making the decision.

\section{EXISTING APPROACH FOR OBJECT DETECTION}

\subsection{Edge Matching}

Digital image processing is meant for processing digital computer. It is the use of computer algorithm to perform image processing on digital images. It is a technology widely used for digital image operations like feature extraction, pattern recognition, segmentation, image morphology etc. This is the developed field of image processing. Edge detection is basically image segmentation technique, divides spatial domain, on which the image is defined, into meaningful parts or regions. Uses edge detection techniques, such as the Canny Edge Detection, to find edges. Changes in lighting and color usually don't have much effect on image edges [7].

\subsection{The Viola-Jones Object Detection Framework}

The significant features of this algorithm are as follow -

1. Robust - This comprises the high rate of detection (high True-Positive Rate \& very low False-Positive Rate ).

2. RealTime- This comprises the ability of processing 2 frames per second for practical area.

3. Face Detection and not recognition -The aim of this method is to differentiate faces from non faces (The initial step of identification is face detection).

\subsection{Deep Neural Network for Object Detection}

In this technique major aim to detect the class sceptic expandable object detection by anticipating group of bounding boxes, which is the potential representation of object. In exact term, here uses the Deep Neural Network Architecture (DNNA), from which the produced output is fixed number of bounding boxes. Along with this fixed bounding boxes its output a score for each box representing the confidence of box containing an object. $[9,10]$ 


\section{APPLICATION OF OBJECT RECOGNITION}

Here introduced some different applicable areas of object recognition are as follow:

- Android Eyes - Object Recognition

- Image panoramas

- Image watermarking

- Global robot localization

- $\quad$ Face detection

- Optical Character Recognition

- $\quad$ Manufacturing Quality Control

- Content-Based Image Indexing

- Object Counting and Monitoring

- Automated vehicle parking systems

- Visual Positioning and tracking

- Video Stabilization

\section{LITERATURE REVIEW}

In this section we proposed a rotation invariant part based model which helps in identifying the object in high resolution remote sensing images. In particularly the complex shape geospatial object is segmented into different smaller subparts, after this structure information about different subpart is obtained and this information managed with respect to polar coordinate so we have the rotation invariance on object configuration. At the same time we also obtain the pose variance of individual part relative to object. The features of rotated parts and object are encoded, thus introduces a novel rotation invariant model by extending histogram oriented gradients. In last step of detection, the clustering technique is adopted to locate all different parts of the potential object; meanwhile this technique is also adopted to inter-mix the detection outcomes. Thus by following the above methodology an efficient detection model is confronted and evaluated experiment ramification shows the robustness and precision of introduces detection system [1].

Here a new methodology is introduced for object recognition named as Artificial Intelligence. This method of recognition is shape dependent method and work in large circumstances, from different lighting situation to affine transformations. Here major attention is paid on neural node of a network that enables system to learn and identify the objects; earlier system has no information about it. The two significant framework of learning i.e supervised techniques, in arrangement of feed-forward networks with error backpropagation, and unsupervised techniques, in arrangement of a self- organizing map, are adopted that helps in understanding of object in an image. In order to have a non lacking system, complete account is used by the proposed image processing methodology that is responsible for recognition of object in an image. The principle working of these techniques is retrieval of different shapes from an image and creates a descriptor vector that helps to overcome the difficulty of affine transformation. The major goal is to introduce a complex object recognition system. The achievement of above aim to build a system that helps in artificial object recognition forms a good basis for further development [2].The pre-programmed identification of complex shape in high resolution remote sensing image is major challenge confronted in the area of object detection. Here latter, a new principle method of object identification is introduced depend on spatial sparse coding bag-of-words (SSCBOW) model helps in determining the solution of confronted problem. Particularly, a unit for processing is selected with the help of sliding window, and retrieve feature, for encoding of the geometric information a spatial mapping technique is used, this helpful not only in determining the relative position of the different parts of object yet proves useful tool to handle rotation invariance. In contrast with the $\mathrm{K}$ means methodology to encode visual word in primitive BOW model, sparse coding is adopted so that a lower reconstruction error is obtained. Lastly, for identifying targets this proposed SSCBOW model integrated with the linear support vector machine model [3].

The modelling of Human Object Intervention (HOI) is responsible for identifying action of human from a single still image, which deduces the mutual information related to spatial structure between human and operated object along with their appearance. The pre-existing techniques of detection are largely depends on the accurate detection of human and object and computation of human poses; this clearly reflect that they are more reactive to the large change in human pose, occlusion and disagreeable identification of smaller objects. In order to overcome this flaw, a novel impeccable approach is presented in this work. This method studied different spatial pose of object interaction, recognised as probabilistic density function and helpful in recounting the human interaction with manipulated object in distinguished actions. Particularly, a novel framework is introduced with faultless HOI descriptor and constructs a related matching model for robust identification of human action in still image. Altogether, proposed framework is extended to identify HOI particularly in videos; this utilizes the above introduced representation for implicit frame selection to nullify immaterial or noisy frames by temporal structured HOI modelling. The large scale experiments are performed on two image action and video action datasets. The ramification obtained from the above experiment clearly shows the effectiveness and helpfulness in achieving the futuristic performance of our proposed work in contrast with other competitive technique [4]

In area of object recognition and remote sensing in order to match different feature of image and to localize the objects of image Scale Invariant Feature Transform(SIFT) and its different version are largely applied. But because of the presence of granular noise it performance degrade in synthetic aperture radar (SAR) images. The objective of this paper is to confront SIFT-like algorithm particularly devoted towards SAR imaging therefore termed as SAR-SIFT. The working framework of proposed algorithm comprises identification of key-points and evaluation of local descriptor. Thus introduces a novel gradient definition, by producing a magnitude and orientation which proves robust against granular noise. It follow the different action of SIFT algorithm to SAR images. The advancement bring out by this proposed work is studied and compared with the work of the existing approach. Also the application of SAR-SIFT is presented to registration of SAR image by having variation in incidence angle[5]. 


\section{PROPOSED WORK}

After discussing all of the above we land up with following proposed work:

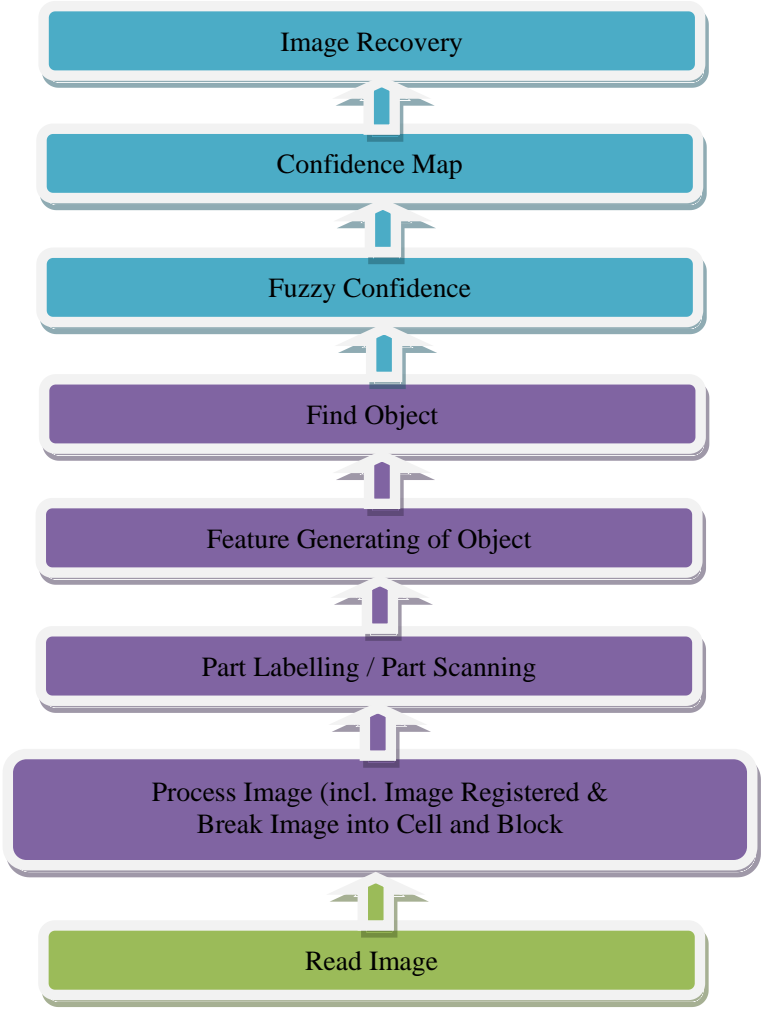

Fig 1: Proposed Work Architecture

Algorithm of Proposed work is mentioned in following figure:

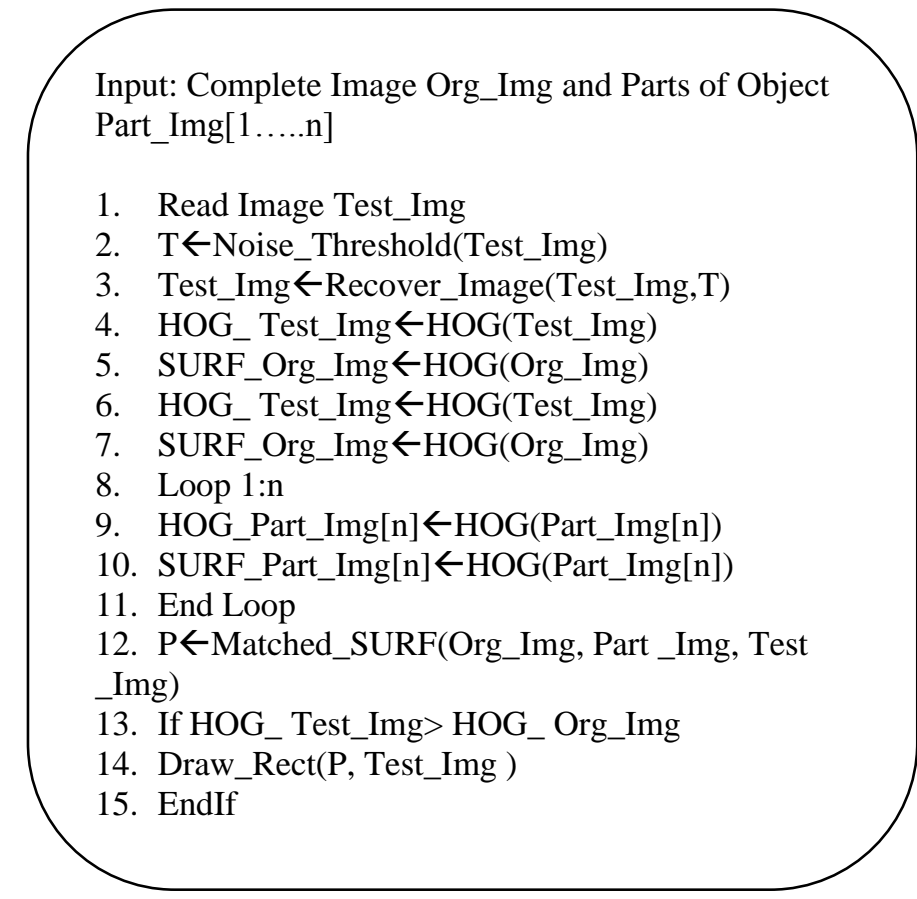

Fig 2: Algorithm of proposed work

\section{SIMULATION AND RESULTS}

All the experiments were conducted on a PC, Dual-Core ( $2.20 \mathrm{GHz}$ ), with $2 \mathrm{~GB}$ of RAM, running a Windows7 operating system and 32-bits operating system.

There are mainly four parameters on which we have evaluate our work.
1. Recovery
2. Precision
3. Recall
4. Fmeasure

Recovery shows the recovery of the corrupted image as compare to original image. In our case, we have taken 4 images. Recovery is evaluated on the scale of $0-1$. This recover evaluation is shown in table 1 . One of corrupted image and recovered images are shown in fig 2 and 3 .

Table 1: Recovery of images

\begin{tabular}{|c|c|}
\hline Image Name & Recovery $(0-1)$ \\
\hline 8_r & 0.862536 \\
\hline 11_r & 0.853641 \\
\hline $\mathbf{2}$ & 0.722548 \\
\hline 12_RECOVERY & 0.75472 \\
\hline
\end{tabular}

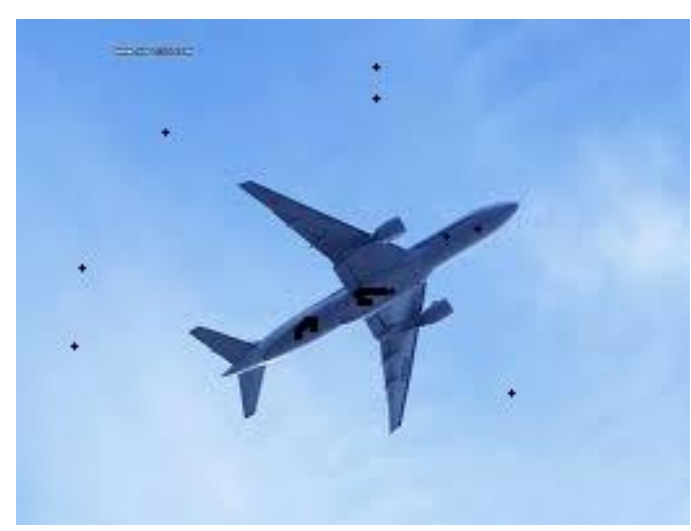

Fig 2: Corrupted Image

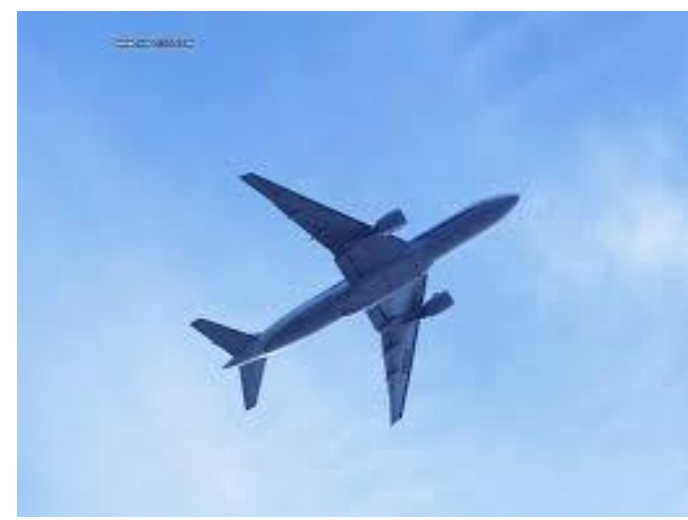

Fig 3: Recovered Image

At the other end, when we evaluate the quality of identify the objects. For identification of object we use precision, recall and fmeasure parameters. The values of these parameters are shown in Table 2. 
Table 2: Quality parameters of object detection

\begin{tabular}{|c|c|c|}
\hline Precision & Recall & F-Measure \\
\hline $\mathbf{0 . 8}$ & 0.66 & 0.726876 \\
\hline
\end{tabular}

\section{CONCLUSION}

There are two aspect of any image. First if it is corrupted image then recover and the other aspect need to find or detect object from the image. In this we have conducted two works:

1. Recovery of image

2. Object detection

From the Table 1 and 2, It is very obvious that the performance of the proposed work is very good in both recovery and object detection as well. We have implemented this work in MATLAB.

\section{REFERENCES}

[1] Purti Singh and DeeptiPandey, "Shape- Based Fish Recognition Using Neural Network", International Journal of Emerging Research in Management \&Technology (Volume-3, Issue-5) May 2014.

[2] Thirumalini.S ,Varunkumar.M and Vanisri, "Detection of Geo-Spatial Targets in High Resolution Remote Sensing Images", International Journal of Computer Science and Information Technologies, Vol. $5(3), 2014$

[3] Matteoli, S and Corsini, G, "Automated Underwater Object Recognition by Means of Fluorescence
LIDAR”, Geoscience and Remote Sensing, IEEE Transactions on (Volume:53, Issue: 1 ) Aug 2014

[4] Flora Dellinger, Julie Delon, YannGousseau, Julien Michel, Florence Tupin, "SAR-SIFT: A SIFT-LIKE ALGORITHM FOR SAR IMAGES", IEEE Transactions on Geoscience and Remote Sensing, jun 2013.

[5] J. Chen, S. Paris, and F. Durand, "Real-Time EdgeAware Image Processing with the Bilateral Grid," ACM Trans. Graphics, vol. 26, no. 3, 2007.

[6] R. C. Gonzalez and R. E. Woods, "Digital Image Processing", Pearson Education, 2002.

[7] Dr.S.Vijayarani and Mrs.M.Vinupriya, "Performance Analysis of Canny and Sobel Edge Detection Algorithms in Image Mining”, International Journal of Innovative Research in Computer and Communication Engineering Vol. 1, Issue 8, October 2013

[8] J.R Jensen, "Introduction to Digital Image Processing" A Remote Sensing Perspective 1996.

[9] C. Szegedy, A. Toshev, and D. Erhan, "Deep neural networks for object detection" In Advances in Neural Information Processing Systems (NIPS), 2013.

[10] Dumitru Erhan, Christian Szegedy, Alexander Toshev, and Dragomir Anguelov, "Scalable Object Detection using Deep Neural Networks", Computer Vision Foundation 2014. 